\title{
Association between serum progesterone levels on the day of frozen-thawed embryo transfer and pregnancy outcomes after artificial endometrial preparation
}

\section{Jing Zhu}

Shanghai Jiao Tong University School of Medicine https://orcid.org/0000-0003-1536-6796

\section{Qianqian Zhu}

Shanghai Jiao Tong University School of Medicine

Jialyu Huang

Shanghai Jiao Tong University School of Medicine

\section{Meiting Qiu}

Shanghai Jiao Tong University School of Medicine

Yanwen Zhu

Shanghai Jiao Tong University School of Medicine

\section{Renfei Cai}

Shanghai Jiao Tong University School of Medicine

Yanping Kuang ( $\square$ kuangyp9hospital@126.com )

\section{Research}

Keywords: progesterone, live birth rate, artificial cycle, frozen embryo transfer, dydrogesterone

Posted Date: August 21st, 2020

DOl: https://doi.org/10.21203/rs.3.rs-16976/v2

License: (c) (i) This work is licensed under a Creative Commons Attribution 4.0 International License.

Read Full License 
The authors have withdrawn this preprint from Research Square 